\title{
Psicosis inducida por cannabis: características clínicas y su diferenciación con la esquizofrenia con y sin consumo de cannabis asociado
}

\section{Cannabis-induced psychosis: clinical characteristics and its differentiation from schizophrenia with and without cannabis use}

\author{
David Rentero*, Francisco Arias*, Sergio Sánchez-Romero**, \\ Gabriel Rubio*,***, Roberto Rodríguez-Jiménez*,***,****. \\ * Servicio de Psiquiatría. Instituto de Investigación Sanitaria Hospital Universitario 12 de Octubre (imas12), Madrid. España. \\ ** Servicio de Psiquiatría. Hospital Universitario Fundación Alcorcón. España. \\ *** Universidad Complutense de Madrid (UCM), Madrid. España. \\ **** Centro de Investigación Biomédica en Red de Salud Mental (CIBERSAM), Madrid. España.
}

\section{Resumen}

El consumo de cannabis se considera un factor de riesgo establecido para el desarrollo de psicosis. Diferenciar los trastornos inducidos por cannabis de la esquizofrenia resulta útil desde el punto de vista pronóstico y terapéutico. Se diferenciaron tres grupos de pacientes hospitalizados: psicosis inducida por cannabis (PIC) $(\mathrm{n}=69$; Media de edad $=27,4, D E=6,5 ; 82,6 \%$ varones $)$, esquizofrenia con abuso o dependencia de cannabis $(\mathrm{EZ}+\mathrm{CB})(\mathrm{n}=57$; Media de edad $=31,9$, $D E=10,1 ; 94,7 \%$ varones) y esquizofrenia sin abuso o dependencia de cannabis (EZ) ( $\mathrm{n}=181$; Media de edad $=41,8, D E=13,3 ; 54,1 \%$ varones). Se utilizó la escala Psychiatric Research Interview for Substance and Mental Disorders (PRISM-IV) para la diferenciación de cuadros inducidos. El grupo PIC presentó puntaciones inferiores en la subescala PANSS negativa $(M=12,9, D E=5,9 ; F=32,24 ; p<0,001)$, menos alucinaciones auditivas $\left(60,3 \% ; \chi^{2}=6,60 ; p=0,037\right)$ y mayor presencia de manía $\left(26,1 \%\right.$ vs. $\left.12,3 \% ; \chi^{2}=32,58 ; p<0,001\right)$ en comparación con el grupo EZ + CB. Hubo pocas diferencias clínicas entre los pacientes con esquizofrenia, independientemente del consumo de cannabis. La edad del primer ingreso por psicosis fue menor en ambos grupos de psicóticos consumidores $(M=26,1, D E=6,4$ en PIC y $M=25,3, D E=$ 6,2 en $\left.\mathrm{EZ}+\mathrm{CB} ; \chi^{2}=20,02 ; p<0,001\right)$. No se observó un patrón clínico característico de las psicosis inducidas por cannabis, aunque sí se demostró el papel precipitante del cannabis en la aparición de psicosis, dada la menor edad de ingreso en los consumidores.

Palabas clave: Psicosis; Esquizofrenia; Cannabis; Psicosis inducidas.

\begin{abstract}
Cannabis use is considered an established risk factor for psychosis development. Differentiating between cannabis-induced disorders and schizophrenia is useful for prognostic and therapeutic purposes. Three inpatients groups were differentiated: cannabis-induced psychosis $(\mathrm{CIP})(\mathrm{n}=69$; mean age $=27.4, S D=6.5 ; 82.6 \%$ males $)$, schizophrenia with cannabis abuse or dependence $(\mathrm{SZ}+\mathrm{CB})(\mathrm{n}=57$; mean age $=31.9, S D=10.1 ; 94.7 \%$ males $)$ and schizophrenia without cannabis abuse or dependence (SZ) (n=181; mean age $=41.8, S D=$ 13.3; 54.1\% males). The Psychiatric Research Interview for Substance and Mental Disorders (PRISM-IV) scale was used to differentiate induced psychosis. The CIP group presented lower mean scores on the negative PANSS subscale $(M=12.9, S D=5.9 ; F=32.24, p<0.001)$, fewer auditory hallucinations $\left(60.3 \% ; \chi^{2}=6.60, p=0.037\right)$ and greater presence of mania $\left(26.1 \%\right.$ vs. $\left.12.3 \% ; \chi^{2}=32.58, p<0.001\right)$ than the $\mathrm{SZ}+\mathrm{CB}$ group. There were few clinical differences between patients with schizophrenia, regardless of previous cannabis use. The age of first admission due to psychosis was lower in both psychotic inpatients groups with cannabis use $(M=26.1, S D=6.4$ in CIP and $M=25.3, S D$ $=6.2$ in $\left.\mathrm{SZ}+\mathrm{CB} ; \chi^{2}=20.02, p<0,001\right)$. A clinical pattern characteristic of cannabis-induced psychosis was not observed, but the precipitating role of cannabis in the appearance of psychotic symptoms was demonstrated, given the lower age of first admission due to psychosis in cannabis user groups.

Keywords: Psychosis; Schizophrenia; Cannabis; Induced psychosis.
\end{abstract}


$\mathrm{E}$ 1 consumo de cannabis es frecuente entre los pacientes con trastornos psicóticos. Más del 25\% de los pacientes con esquizofrenia tienen una dependencia de cannabis concomitante (Koskinen, Löhönen, Koponen, Isohanni y Miettunen, 2010), aunque es frecuente el policonsumo de otras sustancias (Volkow, 2009).

En las últimas décadas, se han llevado a cabo varios estudios de cohortes con el objetivo de investigar la relación entre el consumo de cannabis y la esquizofrenia. Después del estudio inicial de Andréasson, Allebeck, Engström y Rydberg (1987), se han realizado otros estudios de cohortes con datos consistentes entre ellos. En general, la mayoría de los autores consideran que el consumo de cannabis podría ser un factor de riesgo para el desarrollo de esquizofrenia en sujetos vulnerables, sobre todo cuando el consumo se produce en edades tempranas y en grandes cantidades (Konings, Henquet, Maharajh, Hutchinson y Van os, 2008; Marconi, Di Forti, Lewis, Murray y Vassos, 2016). También se ha observado que este riesgo es mayor que con otras drogas y que es la única droga con la que se ha comprobado un adelanto en la edad de inicio de la psicosis (Large, Sharma, Compton, Slade y Nielssen, 2011). Sin embargo, otros autores han sugerido que, aunque el consumo de cannabis precede a la aparición de los síntomas psicóticos, existiría ya una mayor susceptibilidad para la dependencia de cannabis en sujetos que son vulnerables para el desarrollo de psicosis (Power et al., 2014).

Diversos estudios han demostrado que, en voluntarios sanos, el cannabis produce no solo síntomas positivos sino también síntomas negativos y cognitivos (García-Álvarez, Gomar, García-Portilla y Bobes, 2019), imitando así las características típicas de la esquizofrenia (D'Souza et al., 2004). La psicosis inducida por cannabis supone un estado psicótico cuya resolución se produce antes de un mes, necesitándose para ello tratamiento antipsicótico junto con la abstinencia en el consumo. Los nuevos hallazgos sugieren que una gran cantidad de pacientes con psicosis inducida por cannabis posteriormente desarrollarán afecciones psicóticas crónicas, en alrededor del $50 \%$ de los casos (Starzer, Nordentoft y Hjorthøj, 2018). Apoyando el papel causal del cannabis, el uso de cannabis con mayor potencia, medida por la cantidad de tetrahidrocannabinol (THC), ha demostrado presentar mayor riesgo de producir psicosis (Pierre, Gandal y Son, 2016). Asimismo, existen estudios recientes que han demostrado la implicación del consumo de cannabinoides sintéticos en la aparición de síntomas psicóticos transitorios (Monte et al., 2017), psicosis inducidas (Barratt, Cakic y Lenton, 2013), primeros episodios psicóticos (PEP) (Khan, Pace, Truong, Gordon y Moukaddam, 2016) y recaídas psicóticas en pacientes con esquizofrenia (Celofiga, Koprivsek y Klavz, 2014).
Considerando que la esquizofrenia es una enfermedad del neurodesarrollo y la evidencia existente de que el sistema endocannabinoide modula dicho proceso cerebral (proliferación celular, neurogénesis, migración neuronal, proyecciones axonales), el consumo de cannabis, especialmente en edades tempranas de la vida, podría interferir en el neurodesarrollo, constituyendo una explicación biológica plausible (Lubman, Cheetham y Yücel, 2015). Keshavan (1999) propuso la integración de tres modelos neurobiológicos sobre la patogénesis de la esquizofrenia: el modelo de desarrollo temprano, de desarrollo tardío y el modelo neurodegenerativo. Es probable que la vulnerabilidad premórbida a la esquizofrenia sea causada por una interacción de múltiples factores genéticos y ambientales que afectan el desarrollo cerebral temprano. La aparición del trastorno en la adolescencia puede estar determinada por los procesos de maduración cerebral tardía, así como por el estrés exclusivo de la adolescencia y el impacto de la exposición repetida a estresores neuroquímicos o de tipo ambiental, como puede ser el consumo de drogas (Keshavan, Gilbert y Diwadkar, 2006).

Algunos autores sugieren que la aparición de cuadros psicóticos en pacientes con consumo de cannabis tiene mecanismos patogénicos distintos a la aparición de la psicosis en no consumidores. La acción del THC sobre el sistema cannabinoide, intacto o ya previamente alterado, podría producir alteraciones neurobiológicas distintas a la esquizofrenia en no consumidores, lo que podría repercutir en distintas manifestaciones clínicas (Murray et al., 2017). Por otro lado, también los receptores cannabinoides CB2 y los mecanismos neuroinflamatorios pueden ser relevantes y podrían tener un papel diferencial entre consumidores y no consumidores (Minichino et al., 2019; Suárez-Pinilla, López-Gil y Crespo-Facorro, 2014). Para otros autores, aunque se llegue al desarrollo de psicosis por distintos mecanismos, se produciría una alteración final común en el sistema NMDA, íntimamente regulado por los receptores cannabinoides CB1 (Sánchez-Blázquez, Rodríguez-Muñoz y Garzón, 2014).

Es controvertido si el consumo de cannabis modifica la presentación clínica de la psicosis y si constituye una entidad clínica diferente. De esta forma, se ha intentado establecer una clínica diferencial entre los cuadros psicóticos que aparecen en los consumidores de cannabis y los no consumidores, lo que podría orientar para el diagnóstico. Los datos son bastante discrepantes. El consumo de cannabis ha sido ligado a mayor gravedad de síntomas positivos y menos gravedad de síntomas negativos (Pencer y Addington, 2003). En pacientes ingresados observaron la presencia de más síntomas neuróticos y más síntomas depresivos en las psicosis inducidas (Rubio et al., 2012; Thompson et al., 2016). Otros autores refieren más hostilidad y síntomas ansiosos en psicosis inducidas por drogas versus psicosis primarias (Fraser, Hides, Philips, Proctor y 
Lubman, 2012) o más frecuencia de manía y alteraciones conductuales, aunque los síntomas positivos remitían más rápidamente y los síntomas negativos eran menos prominentes (Dawe, Geppert, Occhipinti y Kingswell, 2011). Por otro lado, otros autores no observan diferencias clínicas ni diferencias en los antecedentes familiares entre esquizofrenias ya instauradas, según tuvieran o no historia de consumo de cannabis previo (O'Connell, Sunwoo, McGorry y O'Donoghue, 2019). Es decir, parece que puede haber algunas diferencias clínicas en las psicosis inducidas, pero cuando la esquizofrenia está establecida existen pocas diferencias según haya consumo o no de cannabis, por lo que es importante tener en consideración esa diferencia (Mauri, Di Pace, Reggiori, Paletta y Colasanti, 2017).

En general, los estudios realizados hasta la fecha son bastante contradictorios, probablemente por la heterogeneidad de los criterios de inclusión y exclusión seleccionados. En concreto, la exclusión del abuso o dependencia de otras drogas distintas al cannabis no se ha tenido en cuenta en los trabajos revisados hasta la fecha, algo que podría interferir en los resultados. Además, tampoco existen estudios que hagan una comparativa directa entre pacientes con psicosis inducidas por cannabis, pacientes con esquizofrenia con abuso o dependencia de cannabis y pacientes con esquizofrenia sin criterios de abuso o dependencia de dicha sustancia.

El objetivo del presente estudio fue analizar la posible existencia de diferencias sociodemográficas, clínicas, evolutivas y pronósticas entre tres grupos de pacientes hospitalizados: pacientes con psicosis inducida por cannabis, esquizofrenia con antecedentes de abuso o dependencia de cannabis y esquizofrenia sin antecedentes de abuso o dependencia de cannabis. De cada uno de ellos se estudiaron: 1) características sociodemográficas, antecedentes familiares y antecedentes médicos; 2) características clínicas; 3) consumo comórbido de sustancias; 4) edad del primer ingreso por síntomas psicóticos ajustado por sexo.

\section{Método}

\section{Participantes}

Se reclutaron un total de 331 pacientes. Los criterios de inclusión del presente trabajo fueron: a) pacientes mayores de 18 años; y b) con criterios para el diagnóstico de esquizofrenia $\mathrm{u}$ otros trastornos psicóticos no especificados, según los criterios del DSM-IV-TR (Diagnostic and Statistical Manual of Mental Disorders, Fourth Edition, text rev.) (American Psychiatric Association, 2000). Los criterios de exclusión fueron: a) presencia de psicosis en contexto de trastornos afectivos; b) antecedentes de traumatismo craneoencefálico moderado-grave; c) diagnóstico previo de retraso mental; y d) antecedentes de abuso o dependencia de otras drogas, excepto cannabis y tabaco.

Se llevó a cabo un muestreo intencional y los pacientes fueron clasificados en pacientes con trastorno psicótico e historia de abuso o dependencia de cannabis y pacientes con diagnóstico de esquizofrenia sin abuso o dependencia de drogas, excepto tabaco (EZ). Los pacientes con psicosis y abuso o dependencia de cannabis se subdividieron en esquizofrenia con abuso o dependencia de cannabis (EZ+CB) y psicosis inducidas por cannabis (PIC). Durante las evaluaciones, se produjeron pérdidas de casos (ver Figura 1), antes de su clasificación en grupos y tras ella, ya que los pacientes declinaron su consentimiento para el estudio durante el ingreso.

Según se observa en la Tabla 1, la media de edad de la muestra fue de 36,7 años $(D E=13,1)$, con mayor porcentaje de hombres $(68,1 \%)$. La mayoría de los pacientes eran solteros $(71 \%)$ y convivían con la familia de origen $(59 \%)$. En la comparación entre los tres grupos, la edad basal no se distribuyó de manera equitativa entre los tres grupos $(p<0,001)$. Los pacientes del grupo EZ eran más mayores con respecto al resto de grupos, de manera estadísticamente significativa $(p<0,001)$. Dentro de los pacientes consumidores de cannabis, el grupo EZ+CB eran mayores, si bien esta diferencia no alcanzó una significación estadís-

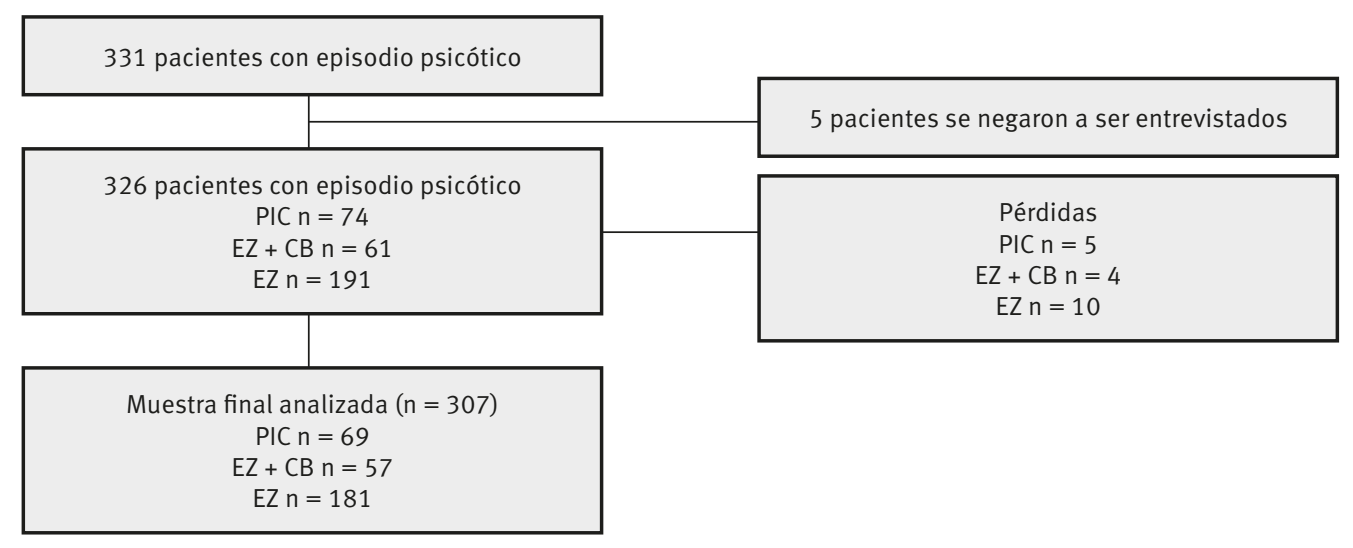

Figura 1. Proceso de selección de la muestra.

PIC: psicosis inducida por cannabis; EZ: pacientes con esquizofrenia; CB: pacientes con abuso o dependencia de cannabis. 
Tabla 1. Características sociodemográficas, antecedentes familiares y antecedentes médicos.

\begin{tabular}{|c|c|c|c|c|c|}
\hline & $\begin{array}{c}\text { Total } \\
(n=307)\end{array}$ & $\begin{array}{c}\text { PIC } \\
(n=69)\end{array}$ & $\begin{array}{c}E Z+C B \\
(n=57)\end{array}$ & $\begin{array}{c}E Z \\
(n=181)\end{array}$ & Valor de la prueba \\
\hline $\begin{array}{l}\text { Edad (años)a } \\
\text { Media (DE) }\end{array}$ & $36,7(13,1)$ & $27,4(6,5)$ & $31,9(10,1)$ & $41,8(13,3)$ & $\begin{array}{c}\chi^{2}=76,61 \\
p<0,001^{\star \star \star}\end{array}$ \\
\hline \multicolumn{6}{|l|}{ Sexo } \\
\hline Masculino & $209(68,1 \%)$ & $\begin{array}{c}57(82,6 \%) \\
\text { R.C }=2,9\end{array}$ & $\begin{array}{c}54(94,7 \%) \\
\text { R.C }=4,8\end{array}$ & $\begin{array}{c}98(54,1 \%) \\
\text { R.C }=-6,3\end{array}$ & \multirow{2}{*}{$\begin{array}{c}\chi^{2}=41,51 \\
p<0,001^{\star \star \star}\end{array}$} \\
\hline Femenino & $98(31,9 \%)$ & $\begin{array}{c}12(17,4 \%) \\
\text { R.C }=-2,9\end{array}$ & $\begin{array}{c}3(5,3 \%) \\
\text { R.C }=-4,8\end{array}$ & $\begin{array}{c}83(45,9 \%) \\
\text { R.C }=6,3\end{array}$ & \\
\hline \multicolumn{6}{|l|}{ Estado civil } \\
\hline Soltero & $218(71 \%)$ & $57(82,6 \%)$ & $45(78,9 \%)$ & $116(64,1 \%)$ & \multirow{3}{*}{$\begin{array}{c}\chi^{2}=11,23 \\
p=0,024^{\star} \\
\text { T apro }=2,84\end{array}$} \\
\hline Casado & $56(18,2 \%)$ & $8(11,6 \%)$ & $6(10,5 \%)$ & $42(23,2 \%)$ & \\
\hline Otros & $33(10,7 \%)$ & $4(5,8 \%)$ & $6(10,5 \%)$ & $23(12,7 \%)$ & \\
\hline \multicolumn{6}{|l|}{ Convivencia } \\
\hline Familia origen & $181(59 \%)$ & $52(75,4 \%)$ & $42(73,7 \%)$ & $87(48,1 \%)$ & \multirow{3}{*}{$\begin{array}{c}\chi^{2}=23,7 \\
p=0,003^{\star \star} \\
\text { T apro }=3,64\end{array}$} \\
\hline Familia propia & $64(20,8 \%)$ & $9(13 \%)$ & $7(12,3 \%)$ & $48(26,5 \%)$ & \\
\hline Otra & 62 (20,2\%) & $8(11,6 \%)$ & $8(14 \%)$ & $46(25,5 \%)$ & \\
\hline \multicolumn{6}{|l|}{ Nivel estudios } \\
\hline Primario & $192(62,5 \%)$ & $36(52,2 \%)$ & $40(70,2 \%)$ & $121(66,9 \%)$ & \multirow{3}{*}{$\begin{array}{c}\chi^{2}=17,01 \\
p=0,009^{\star *} \\
\text { T apro }=-1,33\end{array}$} \\
\hline Secundario & $80(26,1 \%)$ & $27(39,1 \%)$ & $15(26,3 \%)$ & $38(21 \%)$ & \\
\hline Universitario & $30(9,8 \%)$ & $6(8,7 \%)$ & $2(3,5 \%)$ & $22(12,2 \%)$ & \\
\hline \multicolumn{6}{|l|}{ Situación laboral } \\
\hline Paro & 88 (28,7\%) & $35(50,7 \%)$ & 17 (29,8\%) & $36(19,9 \%)$ & \multirow{4}{*}{$\begin{array}{c}\chi^{2}=60,00 \\
p<0,001^{\star \star \star} \\
\text { T apro }=2,43\end{array}$} \\
\hline Activo & $78(25,4 \%)$ & $19(27,5 \%)$ & $12(21,1 \%)$ & $47(26 \%)$ & \\
\hline ILP & $83(27 \%)$ & $1(1,4 \%)$ & $12(21,1 \%)$ & $70(38,7 \%)$ & \\
\hline Otros & 58 (18,9\%) & $14(20,2 \%)$ & $16(28,1 \%)$ & $28(15,5 \%)$ & \\
\hline Hiperactividad infantil & $46(15 \%)$ & $25(36,2 \%)$ & $16(28,6 \%)$ & $5(3,4 \%)$ & $\begin{array}{c}\chi^{2}=42,84 \\
p<0,001^{\star \star \star}\end{array}$ \\
\hline \multicolumn{6}{|l|}{ Antecedentes Familiares } \\
\hline Dependencia drogas & $50(16,3 \%)$ & $19(27,5 \%)$ & $11(19,3 \%)$ & $20(11 \%)$ & $\begin{array}{c}\chi^{2}=10,42 \\
p=0,005^{\star \star}\end{array}$ \\
\hline Psicóticos & $67(21,8 \%)$ & $12(17,4 \%)$ & $15(26,3 \%)$ & $40(22,1 \%)$ & $\begin{array}{c}\chi^{2}=1,47 \\
p=0,47\end{array}$ \\
\hline Comorbilidad médica & $79(26,1 \%)$ & $11(17,3 \%)$ & $10(15,6 \%)$ & $58(32,2 \%)$ & $\begin{array}{c}\chi^{2}=27,84 \\
p=0,26\end{array}$ \\
\hline $\begin{array}{l}\text { IMC basal } \\
\text { Media (DE) }\end{array}$ & $26,6(6,0)$ & $23,4(3,6)$ & $25,1(3,8)$ & $28,3(6,7)$ & $\begin{array}{c}F=10,09 \\
p<0,001^{\star * \star}\end{array}$ \\
\hline
\end{tabular}

Nota. $D E=$ desviación estándar. $\chi^{2}=$ chi-cuadrado. ILP = incapacidad laboral permanente. IMC= índice de masa corporal. $F=$ valor de ANOVA.

T apro = T aproximada, empleando el error típico asintónico basado en la hipótesis nula. R.C. = residuos corregidos (aKruskal- Wallis).

${ }^{\star}$ valores significativos $p<0,05 ;{ }^{\star \star}$ valores muy significativos $p<0,01 ;{ }^{\star \star *}$ valores altamente significativos $p<0,001$

tica. En cuanto al género, el grupo EZ tenía menor prevalencia de varones de manera estadísticamente significativa $(p<0,001)$. Al comparar ambos grupos de consumidores, el grupo EZ+CB presentó un porcentaje mayor de varones $(94,7 \%)$, con significación estadística $(p=0,036)$. En el resto de variables, el grupo EZ convivía con mayor frecuencia con familia propia, presentaba mayor nivel educativo y menor historia familiar de TUS. Además, tenía mayor frecuencia de patología médica (principalmente hipertensión arterial y diabetes), mayor IMC (Índice de Masa Corporal) y menos frecuencia de antecedentes de trastorno por déficit de atención e hiperactividad.

Se obtuvo consentimiento informado por escrito de cada participante, una vez hubieron recibido una descripción completa del estudio. Si los pacientes estaban incapacitados para la toma de decisiones se informó a un familiar. 
El protocolo de investigación fue aprobado por el Comité Ético de Investigación Clínica (CEIC) del Hospital Universitario Fundación Alcorcón.

\section{Instrumentos}

Escala PANSS (Positive and Negative Syndrome Scale). La Escala de los Síndromes Positivo y Negativo desarrollada por Kay, Fiszbein y Opler (1987) y adaptada al español por Peralta y Cuesta (1994), es uno de los instrumentos más utilizados para valorar la sintomatología en pacientes con esquizofrenia. Se trata de una escala heteroaplicada que se cumplimenta a partir de una entrevista semiestructurada de unos 45 minutos de duración. En su versión original, la escala PANSS está compuesta por 30 ítems agrupados en tres factores: síndrome positivo (compuesto por 7 ítems), síndrome negativo (también formado por 7 ítems) y psicopatología general (compuesto por 16 ítems). Las puntuaciones de cada ítem oscilan en un rango de 1 (ausente), 2 (límite con la normalidad), 3 (leve), 4 (moderado), 5 (moderado/severo), 6 (severo) y 7 (extremadamente grave). Las principales propiedades psicométricas de la escala PANSS están actualmente bien documentadas (Kay, Opler y Lindenmayer, 1989; Kay y Sevy, 1990). Wallwork, Fortgang, Hashimoto, Weinberger y Dickinson (2012) propusieron un modelo de cinco factores de la escala, comúnmente etiquetados como «positivo», «negativo», «cognitivo», «depresivo» y «excitativo». En un estudio español, la consistencia interna para el modelo de cinco factores varió de 0.59 (factor excitado) a 0.90 (factor negativo). Aunque la consistencia interna del factor excitado estaba por debajo del límite ampliamente aceptado de 0.70 , estaba cerca de 0.60, un límite aceptable para escalas breves (Rodriguez-Jimenez et al., 2013).

En el presente trabajo, además de usar las puntuaciones totales de la escala PANSS, se utilizaron las subescalas clásicas (positiva, negativa y psicopatología general). Mención especial requiere la subescala negativa, pues fue la utilizada para cuantificar y comparar los síntomas negativos de los tres grupos de pacientes estudiados.

Escala PRISM-IV (Psychiatric Research Interview for Substance and Mental Disorders for DSM-IV) (Hasin et al., 1996). Se trata de una entrevista semiestructurada con la que muchos trastornos del DSM-IV pueden diagnosticarse de manera fiable y alta validez en personas que abusan de sustancias, incluida la dependencia de sustancias, el trastorno depresivo mayor primario e inducido por sustancias, el trastorno psicótico primario e inducido por sustancias, algunos trastornos de ansiedad primaria, el trastorno disocial de la personalidad y el trastorno límite de la personalidad. Para el presente trabajo se utilizó la versión española (Torrens, Serrano, Astals, Pérez-Domínguez y Martín-Santos, 2004), que ha demostrado ser una entrevista mejor estructurada y más precisa para el diagnóstico de psicosis inducidas por drogas que la versión en español de la SCID-1 (Structured
Clinical Interview for DSM-IV) (First, Spitzer, Gibbon y Williams, 2002).

Además de los diagnósticos previos, de esta escala se recogió la presencia de conductas heteroagresivas y el predominio de humor disfórico, depresivo o maníaco, así como la presencia de conducta suicida.

Esta escala ha demostrado una fiabilidad al menos tan buena para los pacientes que abusan de sustancias como lo han demostrado otras entrevistas para muestras generales (Hasin et al., 1996).

Cuestionario de consumo de sustancias. Para la evaluación del consumo de sustancias se formularon una serie de preguntas ad hoc referidas al consumo de alcohol y consumo de drogas, concretamente: cannabis, cocaína, drogas de diseño y opiáceos. Con relación al consumo de alcohol se recogió información sobre si el participante era no bebedor, bebedor o ex-bebedor. Asimismo, también se recogió información sobre otras sustancias como Cannabis/Marihuana/Hachís, Cocaína, Drogas de diseño/Metanfetaminas/ Éxtasis/LSD y Opiáceos. Para cada una de ellas se solicitó información de la edad de inicio del consumo en años, el tiempo de consumo en meses y los días de consumo en el último mes. Estas preguntas fueron formuladas atendiendo a trabajos previos (Dumas et al., 2002) y escalas previamente validadas (Soto-Brandt et al., 2014). Los pacientes con criterios de abuso o dependencia distinta a cannabis o nicotina, fueron excluidos para el estudio.

Escala Addiction Severity Index (ASI) (McLellan et al., 1992). Se trata de una entrevista semiestructurada, de 45 a 60 minutos de duración, administrada por un clínico o entrevistador capacitado, a lo que se suma otros 10-20 minutos de calificación. Se centra en siete áreas que pueden estar afectadas por el consumo de drogas: salud física, empleo y apoyo financiero, actividad ilegal o criminal, relaciones familiares y sociales, síntomas psiquiátricos y consumo de drogas y alcohol. En cada área se valoran la gravedad de los síntomas y el tratamiento aplicado en los últimos 30 días y a lo largo de la vida.

Las propiedades psicométricas de la escala ASI han sido demostradas en diferentes estudios (Butler, Redondo, Fernandez y Villapiano, 2009; Carise et al., 2001).

\section{Procedimiento del estudio}

El reclutamiento de pacientes se llevó a cabo entre el 1 de enero de 2005 y el 31 de diciembre de 2011 en un hospital terciario de la zona sur de la Comunidad de Madrid (Hospital Universitario Fundación Alcorcón). Dicho hospital atiende un área urbana de unos 200.000 habitantes y un área rural de aproximadamente 50.000 habitantes, siendo el único hospital de referencia para dicha población.

Los pacientes se reclutaron de manera consecutiva según ingresaban por un episodio psicótico en la unidad de hospitalización breve (UHB) de dicho hospital. Las evaluaciones clínicas del estudio se realizaron durante el ingreso, 
por parte de un facultativo especialista en psiquiatría de la UHB, mediante una recogida de datos estandarizada, siendo el único entrevistador del estudio.

Hasta el año 2013, se realizó el seguimiento de algunos pacientes que mantenían contacto con los servicios de salud mental de zona, bien a través del hospital o en consultas externas. Dicho seguimiento se realizó para valorar recaídas y reingresos. El seguimiento de la muestra fue de media 51 meses $(D E=2,1)$, mediana 52 (4-84 meses). En el grupo PIC se realizó el seguimiento de 43 pacientes $(M=$ 40,2 meses, $D E=30,4)$, en el grupo $\mathrm{EZ}+\mathrm{CB}$ el seguimiento fue de 33 pacientes con una media de 58,2 meses $(D E=$ 31,9) y en el grupo de EZ se siguieron a 102 pacientes con una media de 59,3 meses $(D E=31,3)$ (diferencias no estadísticamente significativas respecto al grupo de $\mathrm{EZ}+\mathrm{CB}$ ).

\section{Análisis de datos}

Todos los análisis fueron realizados comparando los pacientes EZ frente a pacientes EZ+CB y pacientes con PIC. Se hicieron comparaciones entre los tres grupos mediante Test de Chi-cuadrado $\left(\chi^{2}\right)$ o la prueba exacta de Fisher $(F)$ para datos categóricos y el análisis de la varianza ANOVA o la prueba de Kruskal-Wallis para los datos continuos, dependiendo de si se cumplían las suposiciones de normalidad y tamaño muestral. Se utilizó la prueba de Bonferroni como estudio post-hoc y comparación múltiple entre los tres grupos clínicos. Se usó el test de Kolmogorov-Smirnov para comprobar la normalidad. Se calculó el tamaño del efecto en las comparaciones entre grupos mediante la $\mathrm{d}$ de Cohen (d). Todos los test fueron bilaterales con una $p<0,05$. Los análisis se llevaron a cabo mediante el programa estadístico SPSS 20.0 (Statistical Package for the Social Sciences, 2011).

\section{Resultados}

\section{Diferencias clínicas}

Como muestra la Tabla 2, el grupo EZ presentaba una mayor edad del primer ingreso por psicosis con respecto al resto de grupos. En el análisis post-hoc, dicha diferencia se constató tanto con el grupo PIC $(t=-3,44 ; p=0,001$; $d=0,48)$ como con el grupo $\mathrm{EZ}+\mathrm{CB}(t=-3,67 ; p<0,001$; $d=0,56)$.

No se obtuvo diferencias entre los grupos en cuanto a las puntuaciones totales de la subescala PANSS positiva. Sin embargo, al comparar los diferentes ítems de la subescala, sí que se obtuvieron diferencias en los ítems de excitación $(p<0,001)$ y hostilidad $(p=0,001)$. En el estudio post-hoc, se comprobó que estas diferencias se producían fundamentalmete entre el grupo PIC y el grupo EZ. En la comparación directa, el grupo PIC presentó mayor puntuación en el ítem de excitación con respecto al grupo EZ $(t=4,64 ; p<0,001 ; d=-0,76)$ pero no con respecto al grupo $\mathrm{EZ}+\mathrm{CB}(p=0,70)$. En cuanto al ítem hostilidad, también se demostró mayor puntuación del grupo PIC con respecto al grupo EZ $(t=3,52 ; p=0,001 ; d=-0,56)$ y del grupo $\mathrm{EZ}+\mathrm{CB}$ con respecto al grupo EZ $(t=2,43 ; p=0,01 ; d=-0,008)$. Por el contrario, el grupo EZ+CB presentó mayor puntuación en la subescala PANSS negativa, diferencia que no se produjo con el grupo EZ $(p=0,54)$, pero sí con el grupo PIC $(t=-8,14 ; p<0,001 ; d=1,22)$.

Por último, en el grupo de EZ había menos frecuencia de humor disfórico $\left(\chi^{2}=12,92 ; p=0,02 ; d=0,13\right)$ y de conductas heteroagresivas $\left(\chi^{2}=23,75 ; p<0,001 ; d=0,25\right)$. Los grupos EZ y EZ+CB tenían mayor frecuencia de alucinaciones auditivas $\left(\chi^{2}=6,60 ; p=0,037 ; d=0,08\right)$, menos frecuencia de estado de ánimo expansivo $\left(\chi^{2}=30,46 ; p<0,001\right.$; $d=0,1)$ y mayor desorganización con respecto al grupo PIC $\left(\chi^{2}=4,34 ; p=0,11 ; d=0,14\right)$.

\section{Diferencias evolutivas}

La Tabla 3 refleja los siguientes resultados: en el grupo EZ y EZ+CB había menor remisión interepisódica y más recaídas durante el seguimiento con respecto al grupo PIC. En el análisis post-hoc, la diferencia en cuanto a las recaídas se produjo entre el grupo $\mathrm{EZ}+\mathrm{CB}$ y el grupo PIC $(t=-2.92 ; p=0,05 ; d=0,59)$. En ambos subgrupos de pacientes con esquizofrenia, no hubo diferencias en el curso evolutivo o porcentaje de recaídas. No había diferencias tampoco en el tiempo de seguimiento.

\section{Diferencias en consumo de drogas}

Con respecto al consumo de drogas (ver Tabla 4), había más consumidores de alcohol en el grupo EZ+CB, pero la edad de consumo habitual fue más tardía. Estos consumían mayor cantidad de tabaco. No había diferencias respecto al consumo de cannabis en comparación con el grupo PIC. En la escala ASI, había mayor gravedad en el área de drogas en los inducidos $(M=6,5 ; D E=1,3$ vs $M=5,2 ; D E=1,8$; $F=6,7, p<0,001 ; d=-0,78)$ y mayor gravedad en el área médica en el grupo $\mathrm{EZ}+\mathrm{CB}(M=1,1 ; D E=0,5$ vs $M=1,6$; $D E=1,3 ; F=27,7, p=0,002 ; d=0,55)$.

En el grupo de EZ había menos consumidores de tabaco, de alcohol y cocaína con respecto al grupo EZ+CB. En la escala ASI había mayor gravedad en las áreas de alcohol $(M=2,2 ; D E=1,5$ vs $M=1,4 ; D E=1,1 ; F=8,6, p<0,001 ; d=$ $-0,7)$, drogas $(M=5,2 ; D E=1,9$ vs $M=1,8 ; D E=1,4 ; F=7,8$, $p<0,001 ; d=-2,22)$ y laboral $(M=5,4 ; D E=1,8$ vs $M=4,3$; $D E=1,9 ; F=6,6, p<0,001 ; d=-0,58)$ en el grupo de EZ+CB.

\section{Edad del primer ingreso ajustada por sexo}

Dado que la edad de ingreso puede estar influida por el sexo se realizó un análisis estratificado. En varones se mantuvo la significación estadística $(F=5,08 ; p=0,007)$ siendo la media de 25,9 $(D E=5,6)$ en el grupo PIC, 25,2 $(D E=$ $5,9)$ en el grupo $\mathrm{EZ}+\mathrm{CB}$ y $28,7(D E=8,5)$ en el grupo EZ. En el estudio post-hoc, se constató una diferencia entre el grupo PIC y EZ ( $t=-2,23 ; p=0,04 ; d=0,37)$ y entre el grupo $\mathrm{EZ}+\mathrm{CB}$ y EZ $(t=-2,94 ; p=0,01 ; d=0,45)$, pero no entre am- 
Tabla 2. Características clínicas.

\begin{tabular}{|c|c|c|c|c|c|}
\hline & $\begin{array}{c}\text { Total } \\
(n=307)\end{array}$ & $\begin{array}{c}\text { PIC } \\
(n=69)\end{array}$ & $\begin{array}{l}E Z+C B \\
(n=57)\end{array}$ & $\begin{array}{c}E Z \\
(n=181)\end{array}$ & Valor de la prueba \\
\hline Edad primer ingreso, Media (DE) & $28,9(9,8)$ & $26,1(6,4)$ & $25,3(6,2)$ & $31,1(11,2)$ & $\begin{array}{c}F=11,56 \\
p<0,001^{\star \star \star}\end{array}$ \\
\hline Número ingresos previos, Media (DE) & $2,6(3,7)$ & $0,6(1)$ & $2,2(2,2)$ & $3,5(4,4)$ & $\begin{array}{c}F=16,56 \\
p<0,001^{\star * *}\end{array}$ \\
\hline $\begin{array}{l}\text { PANSS Positiva } \\
\text { Media (DE) }\end{array}$ & $23,3(7,1)$ & $24,3(5,6)$ & $23,3(7,2)$ & $22,9(7,6)$ & $\begin{array}{c}F=0,81 \\
p=0,445\end{array}$ \\
\hline $\begin{array}{l}\text { PANSS Negativa } \\
\text { Media (DE) }\end{array}$ & $20,4(8,9)$ & $12,9(5,9)$ & $22,1(8,9)$ & $23(8,3)$ & $\begin{array}{c}F=32,24 \\
p<0,001^{\star \star \star}\end{array}$ \\
\hline $\begin{array}{l}\text { Desorganización (pensamiento } \\
\text { y conducta) }\end{array}$ & $69(22,5 \%)$ & $10(14,5 \%)$ & $17(29,8 \%)$ & $42(23,2 \%)$ & $\begin{array}{l}\chi^{2}=4,34 \\
p=0,114\end{array}$ \\
\hline \multicolumn{6}{|l|}{ Suicidio } \\
\hline Gestos & $23(7,5 \%)$ & $5(7,2 \%)$ & $2(3,5 \%)$ & $16(8,8 \%)$ & \multirow[t]{2}{*}{$\begin{array}{l}\chi^{2}=4,53 \\
p=0,605\end{array}$} \\
\hline Tentativas & $22(7,2 \%)$ & $4(5,8 \%)$ & $3(5,3 \%)$ & $15(8,3 \%)$ & \\
\hline \multicolumn{6}{|l|}{ Delirios } \\
\hline Paranoide & $275(89,6 \%)$ & $65(94,2 \%)$ & $50(87,7 \%)$ & $160(88,4 \%)$ & $\begin{array}{l}\chi^{2}=2,06 \\
p=0,357\end{array}$ \\
\hline Referencia & $189(61,6 \%)$ & $48(69,6 \%)$ & $34(59,6 \%)$ & $107(59,1 \%)$ & $\begin{array}{l}\chi^{2}=2,41 \\
p=0,299\end{array}$ \\
\hline Megalomaníaco & $66(21,5 \%)$ & $16(23,2 \%)$ & $15(26,3 \%)$ & $35(19,3 \%)$ & $\begin{array}{l}\chi^{2}=1,40 \\
p=0,496\end{array}$ \\
\hline Místico & 74 (24,1\%) & $17(24,6 \%)$ & $12(21,1 \%)$ & 45 (24,9\%) & $\begin{array}{l}\chi^{2}=4,68 \\
p=0,321\end{array}$ \\
\hline Somático & $23(7,5 \%)$ & $4(5,8 \%)$ & $3(5,3 \%)$ & $16(8,8 \%)$ & $\begin{array}{l}\chi^{2}=1,16 \\
p=0,557\end{array}$ \\
\hline Otros & 95 (30,9\%) & $16(23,2 \%)$ & $15(26,3 \%)$ & 64 (35,4\%) & $\begin{array}{l}\chi^{2}=4,16 \\
p=0,125\end{array}$ \\
\hline \multicolumn{6}{|l|}{ Alucinaciones } \\
\hline Auditivas & 222 (72,3\%) & $\begin{array}{l}41(60,3 \%) \\
\text { R.C }=-2,3\end{array}$ & $\begin{array}{c}43(75,4 \%) \\
\text { R.C }=0,2\end{array}$ & $\begin{array}{l}138(76,2 \%) \\
\text { R.C }=1,7\end{array}$ & $\begin{array}{c}\chi^{2}=6,60 \\
p=0,037^{\star}\end{array}$ \\
\hline Visuales & $20(6,5 \%)$ & $6(8,7 \%)$ & $2(3,5 \%)$ & $12(6,6 \%)$ & $\begin{aligned} \chi^{2} & =1,38 \\
p & =0,5\end{aligned}$ \\
\hline Somáticas & $46(15 \%)$ & $10(14,5 \%)$ & $4(7 \%)$ & $32(17,7 \%)$ & $\begin{array}{l}\chi^{2}=3,88 \\
p=0,143\end{array}$ \\
\hline Otras & $13(4,2 \%)$ & $3(4,3 \%)$ & $1(1,8 \%)$ & $9(5 \%)$ & $\begin{array}{l}\chi^{2}=1.11 \\
p=0,574\end{array}$ \\
\hline \multicolumn{6}{|l|}{ Estado de ánimo predominante } \\
\hline Disforia & 77 (25,1\%) & $\begin{array}{c}23(33,3 \%) \\
\text { R.C }=2,1\end{array}$ & $\begin{array}{c}22(38,6 \%) \\
\text { R.C }=2,3\end{array}$ & $\begin{array}{c}32(17,7 \%) \\
\text { R.C }=3,8\end{array}$ & \multirow{3}{*}{$\begin{array}{c}\chi^{2}=30,46 \\
p<0,001^{\star \star \star}\end{array}$} \\
\hline Depresivo & $65(21,2 \%)$ & $\begin{array}{c}9(13 \%) \\
\text { R.C }=-1,9\end{array}$ & $\begin{array}{c}12(21,1 \%) \\
\text { R.C }=0,0\end{array}$ & $\begin{array}{c}44(24,3 \%) \\
\text { R.C }=1,6\end{array}$ & \\
\hline Mania & $41(13,4 \%)$ & $\begin{array}{c}18(26,1 \%) \\
\text { R.C }=3,1\end{array}$ & $\begin{array}{l}7(12,3 \%) \\
\text { R.C }=0,2\end{array}$ & $\begin{array}{l}16(8,8 \%) \\
\text { R.C }=-2,8\end{array}$ & \\
\hline \multicolumn{6}{|l|}{ Heteroagresividad } \\
\hline Leve & 37 (12,1\%) & $8(11,6 \%)$ & $7(12,3 \%)$ & $22(12,2 \%)$ & \multirow{3}{*}{$\begin{array}{c}\chi^{2}=23,18 \\
p<0,001^{\star \star *}\end{array}$} \\
\hline Moderada & 89 (29\%) & $28(40,6 \%)$ & $20(35,1 \%)$ & $41(22,7 \%)$ & \\
\hline Grave & $17(5,5 \%)$ & $4(5,8 \%)$ & $8(14 \%)$ & $5(2,8 \%)$ & \\
\hline
\end{tabular}

Nota. $\mathrm{DE}=$ desviación estándar. $\chi^{2}=$ chi-cuadrado. $F=$ valor de ANOVA. R.C $=$ residuos corregidos.

${ }^{\star}$ valores significativos $p<0,05 ;{ }^{* *}$ valores muy significativos $p<0,01 ;{ }^{* \star *}$ valores altamente significativos $p<0,001$ 
Tabla 3. Características evolutivas.

\begin{tabular}{|c|c|c|c|c|c|}
\hline & $\begin{array}{c}\text { Total } \\
(n=205)\end{array}$ & $\begin{array}{c}\text { PIC } \\
(n=43)\end{array}$ & $\begin{array}{c}E Z+C B \\
(n=33)\end{array}$ & $\begin{array}{c}E Z \\
(n=102)\end{array}$ & Valor de la prueba \\
\hline Remisión interepisódica & $117(57,1 \%)$ & $43(100 \%)$ & $17(51,5 \%)$ & $57(55,8 \%)$ & $\begin{array}{l}\chi^{2}=49,32 \\
p<0,001^{* * *}\end{array}$ \\
\hline Recaídas & $112(54,6 \%)$ & $\begin{array}{c}13(29,5 \%) \\
\text { R.C }=-3,2\end{array}$ & $\begin{array}{c}25(64,1 \%) \\
\text { R.C }=1,8\end{array}$ & $\begin{array}{c}74(54,4 \%) \\
\text { R.C }=1,2\end{array}$ & $\begin{array}{c}\chi^{2}=11,41 \\
p=0,003^{\star \star}\end{array}$ \\
\hline
\end{tabular}

Nota. $\chi^{2}=$ chi-cuadrado. R.C $=$ residuos corregidos

${ }^{*}$ valores significativos $p<0,05 ;{ }^{* *}$ valores muy significativos $p<0,01 ;{ }^{* * *}$ valores altamente significativos $p<0,001$

Tabla 4. Consumo de drogas.

\begin{tabular}{|c|c|c|c|c|}
\hline & $\begin{array}{c}\text { PIC } \\
(n=69)\end{array}$ & $\begin{array}{l}E Z+C B \\
(n=57)\end{array}$ & $\begin{array}{c}E Z \\
(n=181)\end{array}$ & Valor de la prueba \\
\hline $\begin{array}{l}\text { Cannabis: edad consumo } \\
\text { habitual, Media (DE) }\end{array}$ & $18(5)$ & $16,4(3,4)$ & & $\begin{array}{l}F=1,99 \\
p=0,107\end{array}$ \\
\hline $\begin{array}{l}\text { Cannabis: cigarros día máximo, } \\
\text { Media (DE) }\end{array}$ & $7,7(6,2)$ & $7,2(5,3)$ & & $\begin{array}{l}F=0,82 \\
p=0,697\end{array}$ \\
\hline Tabaco a lo largo de la vida & $65(94,2 \%)$ & $53(93 \%)$ & $102(56,4 \%)$ & $\begin{array}{c}\chi^{2}=50,9 \\
p<0,001^{\star \star \star}\end{array}$ \\
\hline Tabaco: cigarros/día Media (DE) & $19,1(11,8)$ & $24.1(13,9)$ & $25.7(16,2)$ & $\begin{array}{c}F=3,07 \\
p=0,049^{\star}\end{array}$ \\
\hline $\begin{array}{l}\text { Alcohol sin datos de abuso/ } \\
\text { dependencia }\end{array}$ & $22(34,9 \%)$ & $26(50 \%)$ & $28(16,5 \%)$ & $\begin{array}{c}\chi^{2}=25,71 \\
p<0,001^{\star \star \star}\end{array}$ \\
\hline $\begin{array}{l}\text { Alcohol edad uso habitual, } \\
\text { Media (DE) }\end{array}$ & $15,6(1,9)$ & $16,8(3,5)$ & $18,8(3,1)$ & $\begin{array}{l}F=2,85 \\
p=0,073\end{array}$ \\
\hline Alcohol UBE máximo Media (DE) & $2,2(6,4)$ & $3,4(4,7)$ & $0,6(1,6)$ & $\begin{array}{c}F=7,93 \\
p<0,001^{\star \star \star}\end{array}$ \\
\hline $\begin{array}{l}\text { Alcohol días por semana Media } \\
\text { (DE) }\end{array}$ & $2,1(2,4)$ & $3,2(2,7)$ & $1,1(2,1)$ & $\begin{array}{c}F=10,7 \\
p<0,001 * \star *\end{array}$ \\
\hline $\begin{array}{l}\text { Cocaína sin datos de abuso/ } \\
\text { dependencia }\end{array}$ & $7(10.9 \%)$ & $11(20.8 \%)$ & $0(0 \%)$ & $\begin{array}{c}\chi^{2}=33,52 \\
p<0,001^{\star \star *}\end{array}$ \\
\hline $\begin{array}{l}\text { Remisión en el consumo de } \\
\text { drogas }\end{array}$ & $38(55,1 \%)$ & $35(61,4 \%)$ & & $\begin{array}{c}\chi^{2}=86,64 \\
p<0,001^{\star \star \star}\end{array}$ \\
\hline
\end{tabular}

Nota. $D E=$ desviación estándar. $\chi^{2}=$ chi-cuadrado. $F=$ valor de ANOVA. UBE $=$ unidades de bebida estándar.

${ }^{*}$ valores significativos $p<0,05 ;{ }^{* *}$ valores muy significativos $p<0,01 ;{ }^{* *}$ valores altamente significativos $p<0,001$.

bos grupos de consumidores. En mujeres, no se mantuvo la significación estadística, probablemente debido a la escasa representación en el grupo $\mathrm{EZ}+\mathrm{CB}(\mathrm{n}=3)$.

\section{Discusión}

La mayoría de los estudios recientes examinan las diferencias demográficas y clínicas entre dos grupos de pacientes: los pacientes con esquizofrenia consumidores frente a los no consumidores y no consideran si son psicosis inducidas o esquizofrenia ya establecida. En relación a los trabajos que abordan el concepto de psicosis inducida, la mayoría incluyen pacientes con un diagnóstico de psicosis inducidas por sustancias y aquellos con un diagnóstico de esquizofrenia y abuso de sustancias (Caton, Samet y Hasin, 2000; Dawe et al., 2011; Fraser et al., 2012) sin determinar que la psicosis inducida sea por cannabis o por otras drogas. Tan solo dos estudios también consideran una tercera cohorte de pacientes: pacientes con un diagnóstico de esquizofrenia que no tenían abuso o dependencia de otras sustancias (Dragogna et al., 2014; Weibell et al., 2013). De ellos, tan solo el trabajo de Dragogna et al. (2014), habla específicamente de cannabis como sustancia de abuso y de psicosis inducida por cannabis. Además, debido a los criterios de inclusión muestral, los trabajos realizados hasta la fecha han sido heterogéneos respecto a la muestra seleccionada, incluyendo PEP con consumo o no de cannabis, esquizofrenia con antecedentes de consumo de cannabis o no, o comparación entre pacientes crónicos y episodios agudos considerando el consumo o los resultados de un análisis toxicológico.

El concepto utilizado por algunos autores de «psicosis cannábica» implica la presencia de una psicopatología concreta de un subtipo psicótico potencialmente diferente. En una revisión de la literatura donde se seleccionaron ocho estudios, siete de estos observaron, al menos, una diferencia clínica estadísticamente significativa. Por lo que los autores concluyen que no es que no exista la «psicosis cannábica», sólo que desde el punto de vista psicopatológico no es cua- 
litativamente diferente de otras formas de psicosis (Baldacchino et al., 2012). Los datos del presente manuscrito apoyarían esta conclusión, no objetivándose diferencias clínicas relevantes que ayuden a establecer una entidad diferenciada de otras psicosis.

Las diferencias sociodemográficas observadas son las esperables. Los consumidores de cannabis son con más frecuencia varones y jóvenes (Kavanagh et al., 2004). Los datos sobre sexo, estado civil, situación laboral es similar a otros estudios (Dawe et al., 2011).

No se observaron diferencias entre los grupos en los antecedentes familiares de psicosis, apoyando el papel de una vulnerabilidad familiar para el desarrollo de psicosis que puede precipitarse con el consumo de cannabis, lo que lleva a destacar el papel de la prevención del consumo en los sujetos de alto riesgo de desarrollo de psicosis. Igualmente, otros autores han apoyado que los individuos con psicosis inducida por cannabis son genéticamente similares a aquellos con trastornos esquizofrénicos (Wilson, Szigeti, Kearney y Clarke, 2018) y se han descrito elevados porcentajes de antecedentes familiares de psicosis en pacientes con esquizofrenia y consumo de cannabis (Bersani, Orlandi, Kotzalidis y Pancheri, 2002). Esto apoya una posible interacción entre consumo de cannabis y la vulnerabilidad genética a la psicosis en el riesgo incrementado de psicosis de estos pacientes. Sin embargo, sí existían diferencias respecto a los antecedentes familiares de trastornos por uso de sustancias.

Se observó la presencia de menos síntomas negativos en los cuadros inducidos, pero no había diferencias entre los dos grupos de pacientes con esquizofrenia. Uno de los hallazgos referidos de forma más constante en la literatura es la presencia de menos síntomas negativos en consumidores de cannabis y de drogas en general (Baldacchino et al., 2012). De cualquier forma, no es habitual considerar si son inducidos o esquizofrenias o el tiempo de evolución del cuadro. Los datos de este estudio sugieren que, en los trastornos inducidos, efectivamente, existen menos síntomas negativos, al igual que señalan otros autores (Caton et al., 2005), pero cuando se considera la presencia de esquizofrenia, esa diferencia desaparece. Así, en estudios donde solo se han considerado la presencia de esquizofrenia tampoco se observó esa diferencia según el consumo (Boydell et al., 2007).

Durante la fase aguda de la psicosis no se observó una diferencia en la puntuación total de la subescala positiva de la PANSS entre los tres grupos de pacientes, al igual que lo referido por otros autores (Boydell et al., 2007; Stone et al., 2014). Sin embargo, sí se evidenció una mayor puntuación en los ítems de excitación y hostilidad en los inducidos frente a los demás grupos como describen otros autores (Baeza et al., 2009). En el presente trabajo, además se observó que en el ítem hostilidad también existieron diferencias entre ambos grupos de pacientes con esquizofrenia.
Una de las características clínicas que se ha considerado relacionadas con las psicosis inducidas es la menor frecuencia de alucinaciones auditivas (Caton et al., 2005; Drake et al., 2011), que también se observó en este trabajo. También eran menos frecuentes los pacientes con presencia de desorganización en los cuadros inducidos. Sin embargo, no hay diferencias en la desorganización en esquizofrenia con o sin consumo de cannabis. La presencia de conductas heteroagresivas ocurría con mayor frecuencia en ambos grupos de consumidores, algo que se ha descrito igualmente en otros pacientes psicóticos con adicciones (Fraser et al., 2012), por lo que se puede considerar relacionado con la presencia del consumo de drogas. En una muestra de pacientes con esquizofrenia y consumo de cannabis había mayor hostilidad que en los no consumidores (Caspari, 1999).

Respecto al estado de ánimo predominante durante la fase aguda, se observaba más disforia en el grupo de EZ+CB y más síntomas maníacos en los cuadros inducidos. Otra característica que se ha sugerido en los cuadros inducidos es la presencia de un humor expansivo. En otros estudios, se ha objetivado que los consumidores de cannabis tenían más síntomas maniformes (McGuire et al., 1994; Núñez y Gurpegui, 2002; Rottamburg, Ben-Arie, Robins, Teggin y Elk, 1982; Stone et al., 2014;) como se objetiva en este estudio. Una explicación es que la presencia de cuadros psicóticos con un humor expansivo, en no consumidores, lo llevaría a filiarse como psicosis afectiva o trastorno esquizoafectivo. Otros autores observaron que los pacientes con psicosis inducida experimentan síntomas maníacos más graves y conducta disruptiva a su llegada al hospital que aquellos con un trastorno psicótico primario (Dawe et al., 2011). En un estudio reciente, se dividieron dos subtipos de trastornos inducidos por cannabis: psicosis inducida por cannabis y trastorno afectivo inducido por cannabis (Shah, Chand, Bandawar, Benegal y Murthy, 2017), alegando que dicha diferenciación en el momento del diagnóstico puede ser valiosa para predecir el curso de la enfermedad y decidir el plan terapéutico. Sin embargo, la mayoría de pacientes con psicosis inducida por cannabis que evolucionaron a trastornos psicóticos presentaron altos porcentajes de síntomas afectivos.

Diversos autores han señalado que existen pocas diferencias clínicas entre pacientes con psicosis, independientemente del consumo (McGuire et al., 1994). La evaluación transversal del cuadro no permite una diferenciación entre psicosis en consumidores de cannabis y otros cuadros psicóticos. Las escasas diferencias clínicas no permiten establecer un adecuado diagnóstico diferencial a través de la valoración exclusivamente psicopatológica. Las psicosis inducidas pueden ser el estadio inicial de la esquizofrenia, ya que sobre el $50 \%$ de éstas evolucionan a una esquizofrenia (Arendt, Rosenberg, Foldager, Perto y Munk-Jørgensen, 2005; Caton et al., 2007; Mauri et al., 2017; Sara, Burgess, 
Malhi, Whiteford y Hall, 2014; Starzer, et al., 2018) y cuando ésta se establece, la clínica parece indistinguible de la esquizofrenia en no consumidores (Boydell et al., 2007). Por lo tanto, tener en consideración si son psicosis inducidas o no en los estudios clínicos puede explicar parte de las discrepancias observadas.

Otro aspecto a considerar es la presencia de otros trastornos adictivos comórbidos, dado que el policonsumo suele ser habitual. Así, excluyendo los efectos del abuso o dependencia de otras drogas en pacientes con esquizofrenia y dependencia de cannabis, se podrá analizar mucho mejor el impacto que tiene el consumo de cannabis (Dubertret, Bidard, Adès y Gorwood, 2006). Por lo tanto, una fortaleza del presente estudio es la exclusión de criterios de abuso o dependencia de otras drogas distintas al cannabis.

La edad de primer ingreso fue menor en los dos grupos de consumidores, en consonancia con múltiples estudios (Dawe et al., 2011; Van Dijk, Koeter, Hijman, Kahn y Van den Brink, 2012) que sugieren que el consumo de cannabis es, al menos, un factor precipitante de la psicosis. En un metaanálisis se señaló que era la única droga capaz de adelantar la edad de inicio de la psicosis (Large et al., 2011). Debido al efecto de género sobre esta variable, la muestra se estratificó por género. En varones se confirmó la menor edad del primer ingreso en los grupos de consumidores. Se ha referido que el cannabis está asociado a un inicio precoz de los síntomas comparado con otras drogas, especialmente entre las mujeres (Allegri et al., 2013), y las diferencias en la edad de inicio según el sexo es menor en los consumidores de cannabis, aunque otros autores observan un adelanto independiente del sexo (Dekker et al., 2012).

No se observaron diferencias en el curso evolutivo entre los grupos de EZ+CB y EZ. Habitualmente los consumidores presentan más recaídas y mala adherencia terapéutica (Zammit et al., 2008). En varios estudios, el consumo de cannabis no se asoció con diferencias psicopatológicas, pero las recaídas fueron significativamente más altas entre los consumidores (Caspari, 1999; Van Dijk et al., 2012). Posiblemente, el pequeño número de pacientes seguidos en el grupo de EZ+CB no sea capaz de detectar esas diferencias en este estudio.

Este estudio tiene varios puntos fuertes: compara las características sociodemográficas, clínicas, evolutivas y pronósticas entre tres grupos de pacientes (PIC, EZ+CB, EZ), logrando de esta manera diferenciar entre sujetos con psicosis inducidas y pacientes con trastornos psicóticos crónicos. Aunque son pocas las diferencias psicopatológicas entre las psicosis inducidas por cannabis y la esquizofrenia con consumo de cannabis, sí resulta fundamental su diferenciación en los diferentes estudios y en la práctica clínica, dado que en el caso de las primeras el pronóstico es más favorable y el tratamiento antipsicótico puede verse como un tratamiento a corto plazo, enfatizando sobre el tratamiento del abuso o dependencia de drogas. Así, el cese en el consumo de cannabis puede llegar a propiciar, en algunos casos, la remisión completa del cuadro, sin llegar a establecerse un trastorno psicótico crónico que, por otra parte, una vez se establece, parece indistinguible de la esquizofrenia. Además, en el estudio se usaron evaluaciones estandarizadas basadas en el consumo de drogas, aspectos sociodemográficos y psicopatología, así como en criterios diagnósticos operacionales para los diagnósticos clínicos. Finalmente, el tamaño de la muestra permitió analizar la presencia de criterios de abuso o dependencia de cannabis de manera exclusiva, eliminando así el efecto de confusión del abuso o dependencia de otras drogas, aunque no el consumo esporádico de éstas.

Sin embargo, los hallazgos deben ser interpretados teniendo en cuenta ciertas limitaciones metodológicas. Este estudio solo incluyó a pacientes que habían tenido contacto con servicios de psiquiatría, excluyendo todos los pacientes que no lo hicieron, como aquellos que acceden a los servicios de adicciones. Además, los datos sobre el consumo de sustancias se recogieron principalmente de lo reportado por los propios pacientes. No se compararon psicosis inducidas con PEP primarios para intentar diferenciar qué características diferenciales de las psicosis inducidas pueden ser debidas al consumo y cuáles al hecho de tratarse de episodios iniciales, como la presencia de síntomas negativos. Además, no se dispone de datos sobre la persistencia en el consumo durante el seguimiento y se produjo pérdida de pacientes durante el mismo.

En conclusión, fueron pocas las diferencias clínicas del grupo PIC en comparación con el resto de grupos: mayor presencia de alteraciones afectivas de tipo maniforme, menor porcentaje de alucinaciones auditivas y sintomatología negativa y, por último, la presencia de mayor cantidad de conductas agresivas. Sin embargo, estas diferencias clínicas desaparecen cuando la esquizofrenia ya está establecida. Además, los pacientes con dependencia de cannabis tienen una edad de inicio de la psicosis más precoz, sugiriendo, al menos, un papel precipitante de esta sustancia en la aparición de trastornos psicóticos. En futuros estudios, deberían tenerse en consideración la diferenciación entre psicosis inducidas y esquizofrenia con abuso o dependencia de cannabis.

\section{Reconocimientos}

FA estableció los objetivos, diseñó la búsqueda bibliográfica, escribió el primer borrador de este artículo y los consecutivos borradores fueron revisados por DR, SS, RR y GR. FA contribuyó en el diseño completo del estudio y en la interpretación de los datos. FA y DR contribuyeron en definir los procedimientos del análisis de los datos y realizar el análisis estadístico. FA y SS participaron en la recogida de datos. Todos los autores revisaron el borrador y aprobaron la versión final. 


\section{Conflicto de intereses}

El Dr. R. Rodriguez-Jimenez ha sido consultor para, participado en actividades de, o recibido ayudas de: Instituto de Salud Carlos III, Fondo de Investigación Sanitaria (FIS), Centro de Investigación Biomédica en Red de Salud Mental (CIBERSAM), Gobierno Regional de Madrid (S2010/ BMD-2422 AGES; B2017/BMD-3740 AGES CM 2-CM), JanssenCilag, Lundbeck, Otsuka, Pfizer, Ferrer, Juste, Takeda.

El resto de autores declaran no tener ningún conflicto de intereses ni haber recibido ningún tipo de financiación para la realización de este artículo.

\section{Referencias}

Allegri, F., Belvederi, M., Paparelli, A., Marcacci, T., Braca, M., Menchetti, M., ... Tarricone, I. (2013). Current cannabis use and age of psychosis onset: a gender-mediated relationship? Results from an 8-year FEP incidence study in Bologna. Psychiatry Research, 210, 368-370. doi:10.1016/j.psychres.2013.06.010.

APA, American Psychiatric Association (2000). Diagnostic and statistical manual of mental disorders (4th Edition). Washington, DC: American Psychiatric Association.

Andréasson, S., Allebeck, P., Engström, A. y Rydberg, U. (1987). Cannabis and schizophrenia. A longitudinal study of Swedish conscripts. Lancet, 26, 1483-1486.

Arendt, M., Rosenberg, R., Foldager, L., Perto, G. y Munk-Jørgensen, P. (2005). Cannabis-induced psychosis and subsequent schizophrenia-spectrum disorders: follow-up study of 535 incident cases. British Journal of Psychiatry, 187, 510-515.

Baeza, I., Graell, M., Moreno, D., Castro-Fornieles, J., Parellada, M., González-Pinto, A., ... Arango, C. (2009). Cannabis use in children and adolescents with first episode psychosis: influence on psychopathology and short-term outcome (CAFEPS study). Schizophrenia Research, 113, 129-137. doi:10.1016/j.schres.2009.04.005.

Baldacchino, A., Hughes, Z., Kehoe, M., Blair, H., Teh, Y., Windeatt, S. y Crome, I. B. (2012). Cannabis psychosis: examining the evidence for a distinctive psychopathology in a systematic and narrative review. American Journal on Addictions, 21, 88-98. doi:10.1111/j.1521-0391.2012.00295.x.

Barratt, M. J., Cakic, V. y Lenton, S. (2013). Patterns of synthetic cannabinoid use in Australia. Drug and Alcohol Review, 32, 141-146. doi:10.1111/j.1465-3362.2012.00519.x.

Bersani, G., Orlandi, V., Kotzalidis, G. D. y Pancheri, P. (2002). Cannabis and schizophrenia: impact on onset, course, psychopathology and outcomes. European Archives of Psychiatry and Clinical Neuroscience, 252, 86-92.

Boydell, J., Dean, K., Dutta, R., Giouroukou, E., Fearon, P. y Murray, R. (2007). A comparison of symptoms and family history in schizophrenia with and without prior cannabis use: implications for the concept of cannabis psychosis. Schizophrenia Research, 93, 203-210.
Butler, S. F., Redondo, J. P., Fernandez, K. C. y Villapiano, A. (2009). Validation of the Spanish Addiction Severity Index Multimedia Version (S-ASI-MV). Drug Alcohol Dependence, 99, 18-27. doi:10.1016/j.drugalcdep.2008.06.012.

Carise, D., McLellan, A. T., Cacciola, J., Love, M., Cook, T., Bovasso, G. y Lam, V. (2001). Suggested specifications for a standardized Addiction Severity Index database. Journal of Substance Abuse Treatment, 20, 239- 244.

Caspari, D. (1999). Cannabis and schizophrenia: results of a follow-up study. European Archives of Psychiatry and Clinical Neuroscience, 249, 45-49.

Caton, C. L., Drake, R. E., Hasin, D. S., Dominguez, B., Shrout, P. E., Samet, S. y Schanzer, B. (2005). Differences between early-phase primary psychotic disorders with concurrent substance use and substance-induced psychoses. Archives of General Psychiatry, 62, 137-145.

Caton, C. L., Hasin, D. S., Shrout, P. E., Drake, R. E., Dominguez, B., First, M. B., ... Schanzer, B. (2007). Stability of early-phase primary psychotic disorders with concurrent substance use and substance-induced psychosis. British Journal of Psychiatry, 190, 105-111.

Caton, C. L., Samet, S. y Hasin, D. S. (2000). When acute-stage psychosis and substance use co-occur: Differentiating substance-induced and primary psychotic disorders. Journal of Psychiatric Practice, 6, 256-266.

Celofiga, A., Koprivsek, J. y Klavz, J. (2014). Use of synthetic cannabinoids in patients with psychotic disorders: Case series. Journal of Dual Diagnosis, 10, 168-173. doi: 10.1080/15504263.2014.929364.

D’Souza, D. C., Perry, E., MacDougall, L., Ammerman, Y., Cooper, T., Yu-te, W., ... Krystal, J. H. (2004). The psychotomimetic effects of intravenous delta-9-tetrahydrocannabinol in healthy individuals: Implications for psychosis. Neuropsychopharmacology, 29, 1558.

Dawe, S., Geppert, L., Occhipinti, S. y Kingswell, W. (2011). A comparison of the symptoms and short-term clinical course in inpatients with substance-induced psychosis and primary psychosis. Journal of Substance Abuse Treatment, 40, 95-101. doi:10.1016/j.jsat.2010.08.002.

Dekker, N., Meijer, J., Koeter, M., Van den brink, W., Van beveren, N., Kahn, R. S., ... Myin-Germeys, I. (2012). Age at onset of non-affective psychosis in relation to cannabis use, other drug use and gender. Psychological Medicine, 42, 1903-1911. doi:10.1017/S0033291712000062.

Dragogna, F., Mauri, M. C., Marotta, G., Armao, F. T., Brambilla, P. y Altamura, A. C. (2014). Brain metabolism in substance-induced psychosis and schizophrenia: A preliminary PET study. Neuropsychobiology, 70, 195-202. doi:10.1159/000366485.

Drake, R. E., Caton, C. L., Xie, H., Hsu, E., Gorroochurn, P., Samet, S. y Hasin, D. S. (2011). A prospective 2-year study of emergency department patients with early-phase primary psychosis or substance-induced psychosis. American Journal of Psychiatry, 168, 742-748. 
Dubertret, C., Bidard, I., Adès, J. y Gorwood, P. (2006). Lifetime positive symptoms in patients with schizophrenia and cannabis abuse are partially explained by co-morbid addiction. Schizophrenia Research, 86, 284-290. doi:10.1176/appi.ajp.2011.10071051.

Dumas, P., Saoud, M., Bouafia, S., Gutknecht, C., Ecochard, R., Daléry, J.,... d'Amato, T. (2002). Cannabis use correlates with schizotypal personality traits in healthy students. Psychiatry Research, 109, 27-35.

First, M. B, Spitzerm, R. L, Gibbon, M. y Williams, J. B. W. (Eds.) (2002). Structured clinical interview for DSM-IV-TR Axis I disorders (SCID-I). New York, NY: New York State Psychiatric Institute, Biometrics Department.

Fraser, S., Hides, L., Philips, L., Proctor, D. y Lubman, D. I. (2012). Differentiating first episode substance induced and primary psychotic disorders with concurrent substance use in young people. Schizophrenia Research, 136, 110-115. doi:10.1016/j.schres.2012.01.022.

García-Álvarez, L., Gomar, J. J., García-Portilla, M. P. y Bobes, J. (2019). Cannabis use and cognitive impairment in schizophrenia and first-episode psychosis. Adicciones, 31, 89-94. doi:10.20882/adicciones.1328.

Hasin, D. S, Trautman, K. D., Miele, G. M., Samet, S., Smith, M. y Endicott, J. (1996). Psychiatric Research Interview for Substance and Mental Disorders (PRISM): Reliability for substance abusers. American Journal of Psychiatry, 153, 1195-1201.

Kavanagh, D. J., Waghorn, G., Jenner, L., Chant, D. C., Carr, V., Evans, M., ... McGrath, J. J. (2004). Demographic and clinical correlates of comorbid substance use disorders in psychosis: Multivariate analyses from an epidemiological sample. Schizophrenia Research, 66, 115-124.

Kay, S. R., Fiszbein, A. y Opler, L. A. (1987). The positive and negative syndrome scale (PANSS) for schizophrenia. Schizophrenia Bulletin, 13, 261-276.

Kay, S. R., Opler, L. A. y Lindenmayer, J. P. (1989). The Positive and Negative Syndrome Scale (PANSS): Rationale and standardisation. British Journal of Psychiatry, 59-67.

Kay, S. R. y Sevy, S. (1990). Pyramidical model of schizophrenia. Schizophrenia Bulletin, 16, 537-545.

Keshavan, M. S. (1999). Development, disease and degeneration in schizophrenia: A unitary pathophysiological model. Journal of Psychiatric Research, 33, 513-521.

Keshavan, M. S., Gilbert, A. R. y Diwadkar, V. A. (Eds.) (2006). The American Psychiatric Publishing textbook of schizophrenia. Washington, DC: American Psychiatric Publishing.

Khan, M., Pace, L., Truong, A., Gordon, M. y Moukaddam, N. (2016). Catatonia secondary to synthetic cannabinoid use in two patients with no previous psychosis. American Journal on Addictions, 25, 25-27. doi:10.1111/ajad.12318.

Konings, M., Henquet, C., Maharajh, H. D., Hutchinson, G. y Van os, J. (2008). Early exposure to cannabis and risk for psychosis in young adolescents in Trinidad. Acta Psychiatrica Scandinavica, 118, 209-213.
Koskinen, J., Löhönen, J., Koponen, H., Isohanni, M. y Miettunen, J. (2010). Rate of cannabis use disorders in clinical samples of patients with schizophrenia: A meta-analysis. Schizophrenia Bulletin, 36, 1115-1130. doi:10.1093/schbul/sbp031.

Large, M., Sharma, S., Compton, M. T., Slade, T. y Nielssen, O. (2011). Cannabis use and earlier onset of psychosis: A systematic meta-analysis. Archives of General Psychiatry, 68, 555-561. doi:10.1001/archgenpsychiatry.2011.5.

Lubman, D. I., Cheetham, A. y Yücel, M. (2015). Cannabis and adolescent brain development. Pharmacology E Therapeutics, 148, 1-16. doi:10.1016/j.pharmthera.2014.11.009.

Marconi, A., Di Forti, M., Lewis, C. M., Murray, R. M. y Vassos, E. (2016). Meta-analysis of the association between the level of cannabis use and risk of psychosis. Schizophrenia Bulletin, 42, 1262-1269. doi:10.1093/schbul/sbw003.

Mauri, M. C., Di Pace, C., Reggiori, A., Paletta, S. y Colasanti, A. (2017). Primary psychosis with comorbid drug abuse and drug-induced psychosis: Diagnostic and clinical evolution at follow up. Asian Journal of Psychiatry, 29, 117-122. doi:10.1016/j.ajp.2017.04.014.

McGuire, P. K., Jones, P., Harvey, I., Bebbington, P., Toone, B., Lewis, S. y Murray, R. M. (1994). Cannabis and acute psychosis. Schizophrenia Research, 13, 161-167.

McLellan, A. T., Kushner, H., Metzger, D., Peters, R., Smith, I., Grissom, G., ... Argeriou, M. (1992). The Fifth Edition of the Addiction Severity Index. Journal of Substance Abuse Treatment, 9, 199-213.

Minichino, A., Senior, M., Brondino, N., Zhang, S. H., Godwlewska, B. R., Burnet, P. W. J., ... Lennox, B. R. (2019). Measuring disturbance of the endocannabinoid system in psychosis: A systematic review and meta-analysis. JAMA Psychiatry. doi:10.1001/jamapsychiatry.2019.0970.

Monte, A. A., Calello, D. P., Gerona, R. R., Hamad, E., Campleman, S. L., Brent, J., ... Carlson, R. G. (2017). Characteristics and treatment of patients with clinical illness due to synthetic cannabinoid inhalation reported by medical toxicologists: A ToxIC database study. Journal of Medical Toxicology, 13, 146-152. doi:10.1007/s13181-0170605-9.

Murray, R. M., Englund, A., Abi-Dargham, A., Lewis, D. A., Di Forti, M., Davies, C., ... D’Souza, D. C. (2017). Cannabis-associated psychosis: Neural substrate and clinical impact. Neuropharmacology, 124, 89-104. doi:10.1016/j. neuropharm.2017.06.018.

Núñez, L. A. y Gurpegui, M. (2002). Cannabis-induced psychosis: A cross-sectional comparison with acute schizophrenia. Acta Psychiatrica Scandinavica, 105, 173-178.

O’Connell, J., Sunwoo, M., McGorry, P. y O’Donoghue, B. (2019). Characteristics and outcomes of young people with substance induced psychotic disorder. Schizophrenia Research, 206, 257-262. doi:10.1016/j.schres.2018.11.007. Pencer, A. y Addington, J. (2003). Substance use and cog- 
nition in early psychosis. Journal of Psychiatry $\mathcal{E}$ Neuroscience, 28, 48-54.

Peralta, V. y Cuesta, M. J. (1994). Psychometric properties of the positive and negative syndrome scale (PANSS) in schizophrenia. Psychiatry Research, 53, 31-40.

Pierre, J. M., Gandal, M. y Son, M. (2016). Cannabis-induced psychosis associated with high potency "wax dabs". Schizophrenia Research, 172, 211-212. doi:10.1016/j. schres.2016.01.056.

Power, R. A., Verweij, K. J., Zuhair, M., Montgomery, G. W., Henders, A. K., Heath, A. C., ... Martin, N. G. (2014). Genetic predisposition to schizophrenia associated with increased use of cannabis. Molecular Psychiatry, 19, 12011204. doi:10.1038/mp.2014.51.

Rodriguez-Jimenez, R., Bagney, A., Mezquita, L., Martinez-Gras, I., Sanchez-Morla, E. M., Mesa, N., ... Palomo, T. (2013). Cognition and the five-factor model of the positive and negative syndrome scale in schizophrenia. Schizophrenia Research, 143, 77-83. doi:10.1016/j. schres.2012.10.020

Rottamburg, D., Ben-Arie, O., Robins, A. H., Teggin, A. y Elk, R. (1982). Cannabis-associated psychosis with hypomanic features. Lancet, 18, 1364-1366.

Rubio, G., Marín-Lozano, J., Ferre, F., Martínez-Gras, I., Rodriguez-Jimenez, R., Sanz, J., ... Palomo, T. (2012). Psychopathologic differences between cannabis-induced psychoses and recent-onset primary psychoses with abuse of cannabis. Comprehensive Psychiatry, 53, 10631070. doi:10.1016/j.comppsych.2012.04.013.

Sánchez-Blázquez, P., Rodríguez-Muñoz, M. y Garzón, J. (2014). The cannabinoid receptor 1 associates with NMDA receptors to produce glutamatergic hypofunction: Implications in psychosis and schizophrenia. Frontiers in Pharmacology, 4, 169. doi:10.3389/ fphar.2013.00169.

Sara, G. E., Burgess, P. M., Malhi, G. S., Whiteford, H. A. y Hall, W. C. (2014). The impact of cannabis and stimulant disorders on diagnostic stability in psychosis. Journal of Clinical Psychiatry, 75, 349-356. doi:10.4088/ JCP.13m08878.

Shah, D., Chand, P., Bandawar, M., Benegal, V. y Murthy, P. (2017). Cannabis induced psychosis and subsequent psychiatric disorders. Asian Journal of Psychiatry, 30, 180184. doi:10.1016/j.ajp.2017.10.003.

Soto-Brandt, G., Portilla Huidobro, R., Huepe Artigas, D., Rivera-Rei, Á., Escobar, M. J., Salas Guzmán, N.,... Castillo-Carniglia, Á. (2014). Evidencia de validez en Chile del Alcohol, Smoking and Substance Involvement Screening Test (ASSIST). Adicciones, 26, 291-302.

Starzer, M. S. K., Nordentoft, M. y Hjorthøj, C. (2018). Rates and predictors of conversion to schizophrenia or bipolar disorder following substance-induced psychosis. American Journal of Psychiatry, 175, 343-350. doi:10.1176/ appi.ajp.2017.17020223.
Statistical Package for the Social Sciences. (2011). SPSS Base 20.0 User's Guide. Chicago, IL: SPSS Inc.

Stone, J. M., Fisher, H. L., Major, B., Chisholm, B., Woolley, J., Lawrence, J., ... Young, A. H. (2014). Cannabis use and first-episode psychosis: Relationship with manic and psychotic symptoms, and with age at presentation. Psychological Medicine, 44, 499-506. doi:10.1017/ S0033291713000883.

Suárez-Pinilla, P., López-Gil, J. y Crespo-Facorro, B. (2014). Immune system: a posible nexus between cannabinoids and psychosis. Brain, Behavior, and Immunity, 40, 269-282. doi:10.1016/j.bbi.2014.01.018.

Thompson, A., Marwaha, S., Winsper, C., Everard, L., Jones, P. B., Fowler, D., ... Birchwood, M. (2016). Short-term outcome of substance-induced psychotic disorder in a large UK first episode psychosis cohort. Acta Psychiatrica Scandinavica, 134, 321-328. doi:10.1111/acps.12623.

Torrens, M., Serrano, D., Astals, M., Perez-Dominguez, G. y Martin-Santos, R. (2004). Diagnosing comorbid psychiatric disorders in substance abusers: Validity of the Spanish versions of the Psychiatric Research Interview for Substance and Mental Disorders and the Structured Clinical Interview for DSM-IV. American Journal of Psychiatry, 161, 1231-1237.

Van Dijk, D., Koeter, M. W., Hijman, R., Kahn, R. S. y Van den Brink, W. (2012). Effect of cannabis use on the course of schizophrenia in male patients: A prospective cohort study. Schizophrenia Research, 137, 50-57. doi:10.1016/j.schres.2012.01.016.

Volkow, N. D. (2009). Substance use disorders in schizophrenia-clinical implications of comorbidity. Schizophrenia Bulletin, 35, 469-472. doi:10.1093/schbul/ $\operatorname{sbp} 0.16$.

Wallwork, R. S., Fortgang, R., Hashimoto, R., Weinberger, D. R. y Dickinson, D. (2012). Searching for a consensus five-factor model of the Positive and Negative Syndrome Scale for schizophrenia. Schizophrenia Research, 137, 24650. doi:10.1016/j.schres.2012.01.031.

Weibell, M. A., Joa, I., Bramness, J., Johannessen, J. O., McGorry, P. D., Ten Velden Hegelstad, W. y Larsen, T. K. (2013). Treated incidence and baseline characteristics of substance induced psychosis in a Norwegian catchment area. BMC Psychiatry, 13, 319. doi:10.1186/1471244X-13-319.

Wilson, L., Szigeti, A., Kearney, A y Clarke, M. (2018). Clinical characteristics of primary psychotic disorders with concurrent substance abuse and substance-induced psychotic disorders: A systematic review. Schizophrenia Research, 197, 78-86. doi:10.1016/j.schres.2017.11.001.

Zammit, S., Moore, T. H., Lingford-Hughes, A., Barnes, T. R., Jones, P. B., Burke, M. y Lewis, G. (2008). Effects of cannabis use on outcomes of psychotic disorders: Systematic review. British Journal of Psychiatry, 193, 357-363. doi:10.1192/bjp.bp.107.046375. 
